\title{
Novos registros de Ephemerelloidea (Insecta: Ephemeroptera) para o estado de São Paulo
}

\author{
Lucimar Gomes Dias ${ }^{1,4}$, Frederico Falcão Salles ${ }^{2}$, Cleber Macedo Polegatto ${ }^{3}$, \\ Rodolfo Mariano Silva ${ }^{3}$ \& Claudio Gilberto Froehlich ${ }^{3}$
}

Biota Neotropica v7 (n3) - http://www.biotaneotropica.org.br/v7n3/pt/abstract?article + bn00307032007

\author{
Recebido em 15/04/07 \\ Versão reformulada recebida em 21/07/07 \\ Publicado em 01/09/07
}

\begin{abstract}
${ }^{1}$ Museu de Entomologia, Departamento de Biologia Animal, Universidade Federal de Viçosa - UFV, CEP 36571-000, Viçosa, Minas Gerais, Brasil

${ }^{2}$ Centro Universitário Norte do Espírito Santo, Universidade Federal do Espírito Santo - UFES, CEP 29933-480, São Mateus, Espírito Santo, Brasil

${ }^{3}$ Laboratório de Entomologia Aquática, Depto. de Biologia, Setor de Ecologia e Evolução, Faculdade de Filosofia, Ciências e Letras de Ribeirão Preto, Universidade de São Paulo - USP, Av. Bandeirantes, 3900, Bairro Monte Alegre, CEP 14040-901 Ribeirão Preto, SP, Brasil

${ }^{4}$ Autor para correspondência: Lucimar Gomes Dias, e-mail: lucimar.dias@gmail.com
\end{abstract}

\begin{abstract}
Dias, L.G., Salles, F.F., Polegatto, C.M., Silva, R.M. \& Froehlich, C.G. New Records of Ephemerelloidea (Insecta: Ephemeroptera) from São Paulo State. Biota Neotrop. Sep/Dez 2007 vol. 7, no. 3 http://www. biotaneotropica.org.br/v7n3/pt/abstract?article+bn00307032007. ISSN 1676-0603.

Four new records of Ephemerelloidea for São Paulo State, Leptohyphes cornutus Allen, Leptohyphes plaumanni Allen, Tricorythodes bullus Allen, and Traverhyphes (Traverhyphes) indicator (Needham \& Murphy) are presented. The latter species constitutes a new record for Brazil. In addition of the new records, the distribution of the following species are expanded: Leptohyphodes inanis Ulmer, Traverhyphes (Mocoihyphes) edmundsi (Allen), and Traverhyphes (Mocoihyphes) yuati Molineri.

Keywords: aquatic insects, Mayflies, Leptohyphidae, Melanemerellidae.

\section{Resumo}

Dias, L.G., Salles, F.F., Polegatto, C.M., Silva, R.M. \& Froehlich, C.G. Novos Registros de Ephemerelloidea (Insecta: Ephemeroptera) para o Estado de São Paulo. Biota Neotrop. Sep/Dez 2007 vol. 7, no. 3 http://www. biotaneotropica.org.br/v7n3/pt/abstract?article+bn00307032007. ISSN 1676-0603.

Neste trabalho são apresentados quatro novos registros de Ephemerelloidea para o Estado de São Paulo, Leptohyphes cornutus Allen, Leptohyphes plaumanni Allen, Tricorythodes bullus Allen e Traverhyphes (Traverhyphes) indicator (Needham \& Murphy), esta ultima constitui um novo registro para o Brasil. Além dos novos registros, também são ampliadas as áreas de distribuição das espécies Leptohyphodes inanis Ulmer, Traverhyphes (Mocoihyphes) edmundsi (Allen) e Traverhyphes (Mocoihyphes) yuati Molineri, para outros municípios do Estado de São Paulo.
\end{abstract}

Palavras-chave: inseto aquático, Efemerida, Leptohyphidae, Melanemerellidae. 


\section{Introdução}

A ordem Ephemeroptera (Insecta) compreende insetos anfibióticos, com adultos de hábitos terrestres e imaturos aquáticos. Nos últimos anos, o estudo da fauna de Ephemeroptera vem sendo destacado pela utilização destes organismos como bioindicadores em programas de monitoramento da integridade dos ecossistemas aquáticos (Buss et al. 2003, Cummins et al. 2005).

Na América do Sul são conhecidas 14 famílias e aproximadamente 460 espécies de Ephemeroptera (Domínguez et al. 2006). A superfamília Ephemerelloidea na América do Sul está representada por três famílias, Leptohyphidae, Coryphoridae e Melanemerellidae. Coryphoridae e Melanemerellidae são monotípicas, a primeira está reportada para o Brasil, Colômbia e Guiana Francesa e a segunda é endêmica para o Brasil (Ulmer 1920, Peters 1981, Orth et al. 2000, Molineri et al. 2002, Molineri \& Domínguez 2003). Leptohyphidae com uma distribuição Pan-americana é a mais representativa, com atualmente 24 espécies registradas para o Brasil (Molineri 2004, Salles et al. 2004, Dias \& Salles 2005, 2006, Salles \& Molineri 2006).

Na Região Sudeste do Brasil, Ephemerelloidea está constituída por Leptohyphidae e Melanemerellidae, com 14 espécies registradas (Molineri 2001a,b, 2002, 2003, 2004, 2005, Salles et al. 2004, Dias \& Salles 2005, 2006). No Estado de São Paulo um avanço no levantamento da efemeropterofauna tem ocorrido nos últimos anos (Salles et al. 2003, Molineri 2004, 2005). Apesar do recente progresso no conhecimento, uma série de lacunas permanecem por serem preenchidas. Atualmente, apenas cinco espécies de Ephemerelloidea são formalmente conhecidas para esta região: Leptohyphodes inanis Ulmer, Tricorythodes (Mocoihyphes) edmundsi (Allen), T. (M.) yuati Molineri, Tricorythopsis araponga Dias \& Salles e Melanemerella brasiliana Ulmer (Molineri \& Domínguez 2003, Molineri 2004, 2005, Dias \& Salles 2005).

Com o objetivo de dar continuidade no levantamento dos Ephemerelloidea de São Paulo e fornecer subsídios para diversas pesquisas que vêm sendo desenvolvidas com ecologia e sistemática de Ephemeroptera no Brasil, são apresentados novos registros de espécies de Ephemerelloidea para São Paulo. Além disso, são abordados dados sobre taxonomia e distribuição no país de todas as espécies conhecidas para o Estado.

\section{Material e Métodos}

Os exemplares de Ephemerelloidea estudados são provenientes de coletas de campo em rios situados em diferentes municípios de São Paulo (conforme material examinado) e empréstimos de instituições do Estado (Laboratório de Entomologia Aquática, Universidade de São Paulo USP e Laboratório de Entomologia Aquática da Universidade Federal de São Carlos - UFSCar). O material examinado será devolvido às instituições de origem, uma parte será depositada no Museu de Zoologia da Universidade de São Paulo, MZUSP e outra parte no Museu Regional de Entomologia da Universidade Federal de Viçosa, UFVB.

\section{Resultados}

\section{Leptohyphes cornutus Allen}

Comentários: L. cornutus na descrição original tem como localidade-tipo o Estado de Santa Catarina, Brasil (Allen 1967). Em 2002, Molineri ampliou a área de distribuição da espécie, reportando-a para outros dois estados brasileiros, Goiás e Rio de Janeiro, além de Misiones na Argentina. Neste trabalho, a espécie é registrada pela primeira vez para o Estado de São Paulo, Brasil.

Material Examinado: 2 ninfas, São Paulo, Brotas, (-22 17 ' 10"' e $\left.-48^{\circ} 07^{\prime} 32^{\prime \prime}\right), 25 /$ vii/2002, Roque, F. (UFSCar).
Distribuição no Brasil: Santa Catarina: Chapecó, Xanxerê; Goiás: Rio Itiquira; Rio de Janeiro: Nova Friburgo; São Paulo: Brotas.

\section{Leptohyphes plaumanni Allen}

Comentários: L. plaumanni foi originalmente registrada para o Estado de Santa Catarina no Brasil (Allen 1967). Em 2002, Molineri documentou a espécie para o Rio de Janeiro, Brasil, e outras localidades da Argentina. Aqui a espécie L. plaumanni é reportada pela primeira vez para o Estado de São Paulo.

Material Examinado: 17 ninfas, São Paulo, Ribeirão Preto (-21 ${ }^{\circ} 10^{\prime} 39^{\prime}$ ' e $-47^{\circ} 48^{\prime} 37^{\prime}$ '), Mata Madre Santa Teresa, 27/iv/2004, Polegatto, C. M. (7 ninfas USP, 5 ninfas MZUSP e 5 ninfas UFVB); 1 ninfa e 16 subimagos, mesmos dados do primeiro, exceto data 29/i/2004 (USP); 50 subimagos, mesmos dados do primeiro, exceto data 1/iv/2004 (20 subimagos USP, 20 subimagos MZUSP e 10 subimagos UFVB); 5 ninfas, São Paulo, Iporanga (-24 35' 08", e -48 35'35"), Rio Carmo, 11/xi/1993, Froehlich C. G. e Paprocki H. (USP).

Distribuição no Brasil: Santa Catarina: Ipumirin; Rio de Janeiro: Teresópolis; São Paulo: Ribeirão Preto, Iporanga.

\section{Leptohyphodes inanis Ulmer}

Comentários: L. inanis (única representante do gênero) é endêmica para o Brasil, foi oficialmente registrada por Molineri (2005) para o município de Campos do Jordão no Estado de São Paulo. A espécie atualmente conta com somente essa localidade de registro, já que na descrição original só consta "Brasil" (Ulmer 1920). Neste trabalho é feito o segundo registro da espécie para o Estado de São Paulo, município de Salesópolis.

Material Examinado: 2 ninfas, São Paulo, Salesópolis, (-23 31' 56' e $\left.-45^{\circ} 50^{\prime} 47^{\prime \prime}\right)$, Estação Biológica de Boracéia, 22/iii/2001, Froehlich, C. G.; Polegatto, C. M. e Mariano, R.L.S. (1 ninfa USP e 1 ninfa MZUSP).

Distribuição no Brasil: Na descrição original só consta "Brasil"; São Paulo: Campos do Jordão, Salesópolis.

\section{Traverhyphes (Traverhyphes) indicator (Needham \& Murphy)}

Comentários: T. (T.) indicator foi descrita como Leptohyphes indicator, cuja localidade tipo é Misiones, Argentina (Needham \& Murphy 1924). Molineri (2001a) criou um novo gênero para a espécie que passou a ser chamada $T$. indicator. Neste trabalho é feito o primeiro registro da espécie para o Brasil.

Material Examinado: 1 adulto e 30 subimagos: São Paulo, Ribeirão Preto ( $-21^{\circ} 10^{\prime} 39^{\prime \prime}$ e $\left.-47^{\circ} 48^{\prime} 37^{\prime \prime}\right)$, Mata Santa Teresa, 29/i/04, Polegatto, C.M. (1 adulto e 10 subimagos USP, 10 subimagos MZUSP e 10 subimagos UFVB); 42 subimagos: São Paulo, Iporanga (-24 35' 08', -48 35'35'), Parque Estadual Intervales, Córrego Roda d'Agua, 12/xi/92, Froehlich, C.G. e Paprocki, H. (22 subimagos USP, 10 subimagos MZUSP e 10 subimagos UFVB).

Distribuição no Brasil: São Paulo: Ribeirão Preto, Iporanga.

\section{Traverhyphes (Mocoihyphes) edmundsi (Allen)}

Comentários: T. (M.) edmundsi também foi descrita primariamente como integrante do gênero Leptohyphes sendo posteriormente transferida para Traverhyphes (Allen 1973, Molineri 2004). No trabalho original a espécie foi reportada para os três estados que representam a Região Sul do Brasil (Santa Catarina, Paraná e Rio Grande do Sul). Molineri (2004) reportou a espécie para Iporanga e Campos do Jordão no Estado de São Paulo, além de outras localidades na Argentina. Aqui a espécie é documentada para outra localidade de São Paulo, o município de Salesópolis.

Material Examinado: 3 adultos: São Paulo, Salesópolis $\left(-23^{\circ} 31^{\prime} 56^{\prime \prime}\right.$ e $\left.-45^{\circ} 50^{\prime} 47^{\prime \prime}\right)$, Estação Biológica de Boracéia, 
Rio Claro - Poço Verde, 21/iii/01, Froehlich, C.G., Silva Mariano R.L.S., e Polegatto, C.M. (1 adulto USP, 1 adulto MZUSP e 1 adulto UFVB).

Distribuição no Brasil: Rio Grande do Sul: Cachoeira do Sul; Santa Catarina: Joinville, Rio Warnow; Paraná: Morretes, Curitiba, Sete Quedas, Prudentópolis, Guairá; São Paulo: Iporanga, Campos do Jordão, Salesópolis.

\section{Traverhyphes (Mocoihyphes) yuati Molineri}

Comentários: T. (M.) yuati tem como localidade-tipo Misiones na Argentina, no entanto, o autor da espécie no trabalho original também a registrou para o município de Campos do Jordão, São Paulo (Molineri 2004). Neste trabalho, T. (M.) yuati é documentada para outros dois municípios de São Paulo, Salesópolis (Estação Biológica de Boracéia) e Iporanga (Parque Estadual Intervales).

Material Examinado: 1 adulto: São Paulo, Salesópolis (-2331' 56' e -45 50 ' 47'), Estação Biológica de Boracéia, Rio Claro - Poço Verde, 21/iii/2001, Froehlich, C.G., Mariano, R.L.S. e Polegatto, C.M. (USP); 1 adulto: São Paulo, Iporanga ( $-24^{\circ} 35^{\prime} 08^{\prime}$ ' e $\left.-48^{\circ} 35^{\prime} 35^{\prime \prime}\right)$, Parque Estadual Intervales, Cachoeira de Água Comprida, 29/iv/2001 (MZUSP).

Distribuição no Brasil: Rio de Janeiro: Nova Friburgo; São Paulo: Campos do Jordão, Iporanga e Salesópolis.

\section{Tricorythodes bullus Allen}

Comentários: T. bullus foi originalmente registrada para o Estado de Santa Catarina (Allen 1967). Em 2002, Molineri realizou uma revisão do gênero, reportando a espécie para outras localidades da Argentina. Pela primeira vez é feito o registro da espécie para o Estado de São Paulo.

Material Examinado: 5 adultos: São Paulo, Campos do Jordão (-22 $44^{\prime} 22$ " e $-45^{\circ} 35^{\prime}$ 29”'), Parque Estadual Campos do Jordão, 13/vii/2004, Froehlich, C.G., Polegatto, C.M. e Mariano, R.L.S. ( 2 adultos USP, 2 adultos MZUSP e 1 adulto UFVB); 3 ninfas: mesmos dados do anterior, exceto 19/xi/1987, Froehlich, C. G. e Oliveira, L.G. (USP).

Distribuição no Brasil: Santa Catarina: Ipumirim; São Paulo: Campos do Jordão.

\section{Tricorythopsis araponga Dias \& Salles}

Comentários: $T$. araponga no trabalho original foi reportada para Salesópolis, São Paulo (Dias \& Salles 2005).

Distribuição no Brasil: Minas Gerais: Araponga; Rio de Janeiro: Itatiaia; São Paulo: Salesópolis; Espírito Santo: Jerônimo Monteiro.

\section{Tricorythopsis gibbus (Allen)}

Comentários: T. gibbus foi originalmente incluída no gênero Leptohyphes, a localidade-tipo da espécie inclui Santa Catarina e Minas Gerais (Allen 1967). Molineri (2001b) realizou uma revisão do gênero Tricorythopsis no qual incluiu a espécie e ampliou sua distribuição para o município de Campos do Jordão, no Estado de São Paulo.

Distribuição no Brasil: Santa Catarina: Nova Teutônia. Minas Gerais: Ouro Fino. São Paulo: Campos do Jordão.

\section{Melanemerella brasiliana Ulmer}

Comentários: $M$. brasiliana é uma espécie monotípica da família Melanemerellidae e endêmica do Sudeste do Brasil, cuja localidade tipo é o Estado do Espírito Santo (Ulmer 1920). Tratase de uma espécie rara de Ephemerelloidea que por muito tempo teve uma classificação problemática. Esta espécie foi estudada por diversos pesquisadores e passou por três famílias de Ephemeroptera, Ephemerellidae, Tricorythidae e Leptophlebiidae (Ulmer 1920,
Demoulim 1955, Edmunds et al. 1963, Wang \& McCafferty 1996). Em 2003, Molineri \& Dominguez, com o conhecimento da ninfa e do ovo de $M$. brasiliana, e de análises filogenéticas, transferiram o gênero para uma nova família (Melanemerellidae) e ampliaram a área de distribuição da espécie, registrando-a para Campos do Jordão, São Paulo.

Distribuição no Brasil: São Paulo: Campos do Jordão; Espírito Santo (na descrição original não consta o nome do município do ES).

\section{Agradecimentos}

Ao Laboratório de Entomologia Aquática da Universidade Federal de São Carlos, DHB, em especial à Dra. Susana Trivino e ao Dr. Fábio Roque pelo empréstimo de espécimes de São Paulo. Ao CNPQ e FAPESP por prover fundos para Lucimar G. Dias e Rodolfo M. L. Silva, como estudante de Pós-graduação na Universidade Federal de Viçosa e Universidade de São Paulo, respectivamente.

\section{Referências Bibliográficas}

ALLEN, R.K. 1967. New species of New World Leptohyphinae (Ephemeroptera: Tricorythidae). Can. Entomol. 99:350-375.

ALLEN, R.K. 1973. New species of Leptohyphes Eaton (Ephemeroptera: Tricorythidae). The Pan-Pac. Entomol. 49:363-372.

BUSS, D.F., BAPTISTA, D.F. \& NESSIMIAN, J.L. 2003. Bases conceituais para aplicação de biomonitoramento em programas de avaliação de qualidade da água de rios. Cad. de Saúde Pública, Rio de Janeiro: Ensp/Fiocruz. 19:465-473.

CUMMINS, K.W., MERRITT, R.W. \& ANDRADE, P.C.N. 2005. The use of invertebrate functional groups to characterize ecosystem attributes in selected streams and rivers in southeast Brazil. Stud. Neotrop. Fauna Environ. 40:71-90.

DEMOULIN, G. 1955. Melanemerella brasiliana Ulmer, Ephémérellide ou Tricorythide? (Ephemeroptera). Bull. Ann. Soc. Entomol. Belg., 91(7-8):214-216.

DIAS, L.G. \& SALLES, F.F. 2005. Three new species of Tricorythopsis (Ephemeroptera: Leptohyphidae) from Southeastern Brazil. Aquat. Insect. 24:235-241.

DIAS, L.G. \& SALLES, F.F. 2006. A new species of Tricorythodes (Ephemeroptera: Leptohyphidae) from Minas Gerais, Southeastern Brazil. Neotrop. Entomol. 31:56-58.

DOMÍNGUEZ, E., MOLINERI, C., PESCADOR, M., HUBBARD, M.D. \& NIETO, C. 2006. Ephemeroptera of South America In: Adis J, Arias JR, Rueda-Delgado R, Wantzen KM. Aquatic biodiversity in Latin America, Vol. 2. Sofia-Moscow, Pensoft. 642p.

EDMUNDS, G.F.Jr., ALLEN, R.K. \& PETERS, W.L. 1963. An annotated key to the nymphs of the families and subfamilies of mayflies (Ephemeroptera). U. Utah Biol. Ser., 13(1):1-49.

MOLINERI, C. 2001a. Traverhyphes: a new genus of Leptohyphidae for Leptohyphes indicator and related species. Spixiana. 24: 129-140.

MOLINERI, C. 2001b. El género Tricorythopsis (Ephemeroptera: Leptohyphidae): nuevas combinaciones y descripción de nuevas especies y estadios. Rev. Soc. Entomol. Argent. 60:217-238.

MOLINERI, C. 2002. Cladistic analysis of the South American Species of Tricorythodes (Ephemeroptera: Leptohyphidae) with the description of new species and stages. Aquat. Insect. 24:273-308.

MOLINERI, C. 2003. Revision of the South American species of Leptohyphes Eaton (Ephemeroptera: Leptohyphidae) with a key to the nymphs. Stud. Neotrop. Fauna Environ. 38:47-70.

MOLINERI, C. 2004. Phylogeny of the Allenhyphes-Traverhyphes group (Ephemeroptera: Leptohyphidae), with new subgenera, species and combinations. Tijdsch. Entomol. 147:197-220.

MOLINERI, C. 2005. Leptohyphodes inanis (Pictet) and Tricorythodes ocellus Allen \& Roback (Ephemeroptera: Leptohyphidae): new stages and descriptions. Stud. Neotrop. Fauna Environ. 40:247-254. 
MOLINERI, C., PETERS J.G. \& ZÚÑIGA DE CARDOZO, M.C. 2002. A new family Coryphoridae (Ephemeroptera: Ephemerelloidea) and description of winged and egg stages of Coryphorus. Insecta Mundi, 15(2): $117 ; 122$

MOLINERI, C. \& DOMINGUEZ, E. 2003. Nymph and egg of Melanemerella brasiliana (Ephemeroptera: Ephemerelloidea: Melanemerellidae), with comments on its systematic position and the higher classification of Ephemeroptera. J. North. Am. Benthol. Soc. 22:263-275.

NEEDHAM, J.G. \& MURPHY, H.E. 1924. Neotropical Mayflies. Bulletin of the Lloyd Library of Botany, Pharmacy and Materia Medica, No. 24, Entomol. Ser. 4:1-79.

ORTH, K., THOMAS, A., DAUTA, C., HOREAU, V., BROSSE, S. \& ADEMMER, C. 2000. Les Ephémères de la Guyane Française. 1. Premier inventaire générique, a but de biosurveillance (Ephemeroptera). Ephem. 2:25-38.

PETERS, W. L. 1981. Coryphorus aquilus, a new genus and species of Tricorythidae from the Amazon Basin (Ephemeroptera). Aquat. Insect. 3:209-217.
SALLES, F.F. \& MOLINERI, C. 2006. Amanahyphes saguassu: a new genus and species of Leptohyphidae (Ephemeroptera: Ephemerelloidea) from Northern Brazil. Aquat. Insect. 28:1-12.

SALLES, F.F., DA-SILVA, E.R., HUBBARD, M.D. \& SERRÃO, J.E. 2004. As espécies de Ephemeroptera (Insecta) registradas para o Brasil. Biota Neotrop. 4:1-34. http://www.biotaneotropica.org.br/v4n2/pt/abstract?in ventory+BN04004022004

SALLES, F.F., FRANCISCHETTI, C.N., ROQUE, F.O., PEPINELLI, M. \& STRIXINO, S.T. 2003. Levantamento preliminar dos gêneros e espécies de Baetidae (Insecta: Ephemeroptera) do Estado de São Paulo, com ênfase em coletas realizadas em córregos florestados de baixa ordem. Biota Neotrop. 3:1-7. http://www.biotaneotropica.org.br/v3n2/pt/abstract?shortcommunication+BN01103022003

ULMER, G. 1920. Neue Ephemeropteren. Arch. Naturg. 85:1-80.

WANG, T.Q. \& MCCAFFERTY, W.P. 1996. Redescription and reclassification of the South American mayfly Melanemerella brasiliana (Ephemeroptera: Leptophlebiidae). Entomol. News, 107(2):99-103. 